\title{
Refractory Coats' Disease of Adult Onset
}

\author{
D. Beselga A. Campos S. Mendes F. Carvalheira \\ M. Castro D. Castanheira
}

Department of Ophthalmology, Hospital Santo André, Leiria, Portugal

\section{Key Words}

Coats' disease - Adult onset - Macular star - Laser photocoagulation - Photodynamic therapy $\cdot$ Intravitreal bevacizumab

\begin{abstract}
Purpose: We present the case of an 18-year-old Caucasian male with a unilateral macular star and retinal vascular anomalies compatible with adult onset Coats' disease.

Methods: Diagnosis was based on fundoscopic, fluorescein angiography and optical coherence tomography findings.

Results: The patient presented to our emergency department with complaints of low vision in his left eye (LE) detected 10 days before. The best-corrected visual acuity in the LE was 20/50. Fundoscopy of the LE evidenced a complete macular star. Optical coherence tomography showed increased retinal thickness, infiltration of the retinal wall, and detachment of the neuroepithelium. Angiography revealed no appreciable diffusion in the macula. Above the superior temporal (ST) arcade, anomalies in the retinal vasculature were found, with interruption of the peripheral vessels and vessels which were 'sausage'-like. After 1 month, the LE vision evolved to hand movements. Laser photocoagulation was performed in the ST quadrant. Intravitreal injection of bevacizumab $1.25 \mathrm{mg} / 0.05 \mathrm{ml}$ and photodynamic therapy were performed without any significant changes, progression of ST serous detachment of the neuroepithelium, and finally progression to macular fibrosis. Discussion: Coats' disease is usually diagnosed in childhood, but rare cases may occur in adults. Those cases usually have a more indolent course which was not observed in our patient. When there is macular involvement, prognosis is more guarded, despite treatment.
\end{abstract}

\section{Introduction}

In 1908, George Coats first described the retinal vascular abnormalities of Coats' disease [1]. Coats' disease is characterized by abnormalities of the retinal vessels, which include telangiectasia, the formation of aneurysms, and subretinal and intraretinal spots. It commonly occurs in childhood with leukocoria, bad visual acuity, 
and/or strabismus. It mainly affects males in the first decade of life [2]. It has been diagnosed for the first time in adults in rare cases [3]. When this process is detected in adulthood, it usually occurs on one side only and preferably in males. In contrast to childhood onset, many adult patients have no symptoms or exhibit good visual acuity [4]. In adult patients, it must be differentiated from Leber's miliary aneurysms that exhibit a smaller amount of retinal exudation. It can be confused with retinal detachment, toxocariasis, choroidal melanoma, retinitis due to cytomegalovirus or toxoplasmosis [5]. Current treatment options for patients with Coats' disease are ablation of abnormal retinal vessels, either by laser photocoagulation or by cryotherapy, and surgery for retinal detachment. Recently, the dysregulation of vascular endothelial growth factor (VEGF)-mediated angiogenesis was suggested to be a component in Coats' disease [6, 7]. Laser photocoagulation is not an efficient treatment modality if subretinal exudates obscure a target tissue, and photodynamic therapy may be an alternative [6]. The visual prognosis depends on the macular involvement. In some cases, the progression produces retinal detachment, glaucoma, and painful eye that may require enucleation [2].

\section{Case Report}

An 18-year-old Caucasian male presented to our emergency department with complaints of low vision in his left eye (LE), detected in a sports medicine consultation 10 days before. He had a history of cranioencephalic trauma 1 month previously and of a flu-like illness 6 months previously.

The best-corrected visual acuity in the LE was 20/50. There were no changes in the right eye (RE) and best-corrected visual acuity was 20/20. Fundoscopy of the LE evidenced a complete macular star. Optical coherence tomography showed increased retinal thickness, infiltration of the retinal wall, and detachment of the neuroepithelium (fig, 1, left). Angiography revealed no appreciable diffusion in the macula. Above the superior temporal (ST) arcade, anomalies in the retinal vasculature were found, with interruption of the peripheral vessels and vessels which were 'sausage'-like (fig. 2). We excluded possible infectious causes, autoimmune disease, diabetes, hemoglobinopathy, and performed a chest $\mathrm{X}$-ray and brain computed tomography, all of which turned out normal. After 1 month, the LE vision evolved to hand movements. Laser photocoagulation was performed in the ST quadrant. An intravitreal injection of bevacizumab $1.25 \mathrm{mg} / 0.05 \mathrm{ml}$ was given, and photodynamic therapy was performed without any significant changes, progression of the ST serous detachment of the neuroepithelium (fig. 1, middle; fig. 3 ), and finally progression to macular fibrosis (fig. 1, right and fig. 4).

\section{Discussion}

Coats' disease is generally a unilateral condition, characterized by telangiectatic and aneurismal changes of the retinal vessels, associated with large amounts of yellowish subretinal and intraretinal exudate [8]. There are only a few reports of adult cases, and the progression of Coats' disease is relatively slow in older children or adults, and it shows indolent clinical features [9]. According to these characteristics, we consider that our case had an atypical course. The etiology of the disease remains unclear, and several theories have been proposed. Mutations in retinal proteins encoded by CRB1 and NDP are being investigated [10]. In adults, it is important to exclude a history of retinal detachment, diabetes, exposure to radiation, episodes of intraocular inflammation, tapetoretinal degeneration, and vascular occlusion [11]. Regarding treatment, the choice depends on the severity of the disease at presentation. The 
conventional treatment is cryopexy or laser photocoagulation to reduce or eliminate telangiectatic vessels. If successful, it produces reabsorption of exudation. In our case, laser photocoagulation of abnormal vessels was done, but macular star persisted. In 2001, Shields et al. [12] proposed a classification of Coats' disease based on disease course and prognosis: cryotherapy and laser photocoagulation for stage 1-3A, surgical retinal reattachment for most cases of stage 3B, and enucleation for stage 4 .

Photodynamic therapy can also be an alternative to laser photocoagulation by obliterating the multiple abnormal retinal vessels surrounded by the massive retinal exudates [6]. In our case, it had no influence on the final outcome, probably because it was done in an advanced stage of the disease. The use of bevacizumab (Avastin, Genentech) for the treatment of Coats' disease is currently an area of research. The retinal ischemic component in the telangiectatic areas and the neovascularization process show the importance of the angiogenic factors in the disease. There are cases of success with this therapy described in the literature $[2,13]$. In our case, bevacizumab was tried because intraocular VEGF is elevated in Coats' disease and anti-VEGF agents are used to stabilize the blood-retina barrier and reduce the amount of subretinal exudation. Intravitreal triamcinolone could also have been an option to reduce macular edema, and it was considered later on, but the patient refused to have an additional intravitreal injection. Two years after onset, macular star evolved to epiretinal membrane and no additional exudation or glaucoma developed.

\section{Disclosure Statement}

The authors report no proprietary interest or financial support and do not have a conflict of interest.

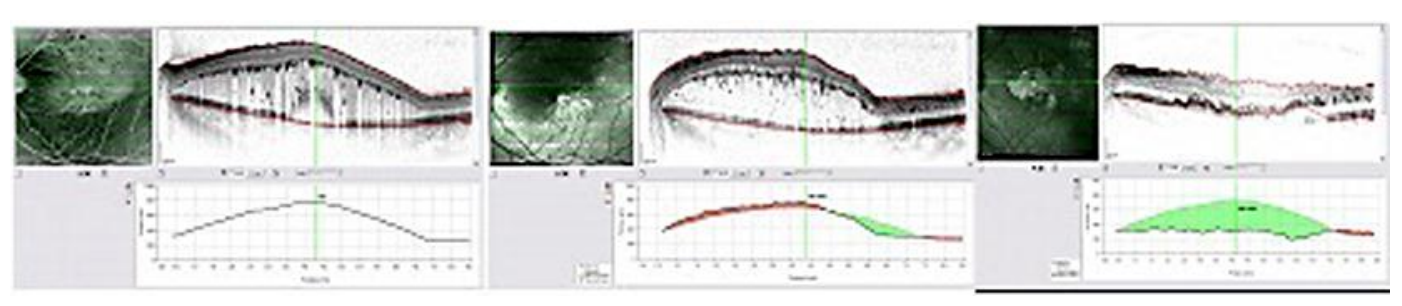

Fig. 1. Optical coherence tomography at presentation (left), 4 months after treatment (middle), and 1 year after treatment (right). 

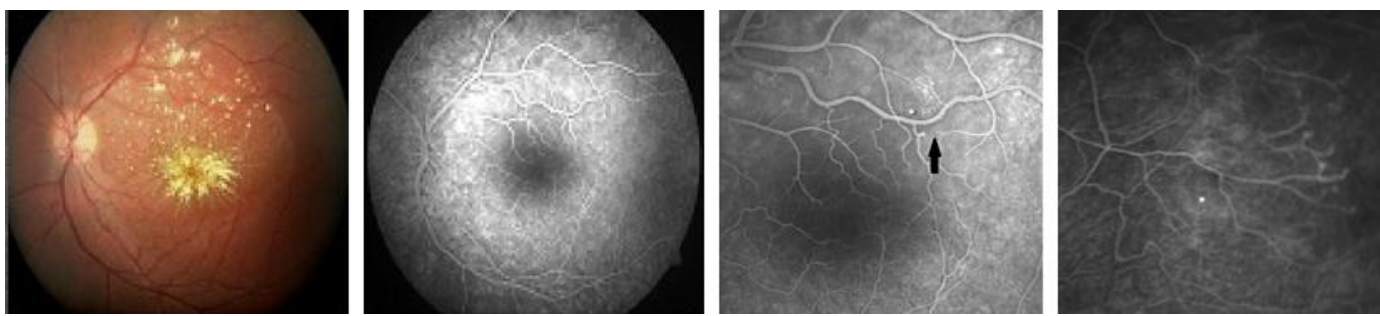

Fig. 2. Fluorescein angiography at presentation with telangiectatic changes of the retinal vessels in the temporal periphery.
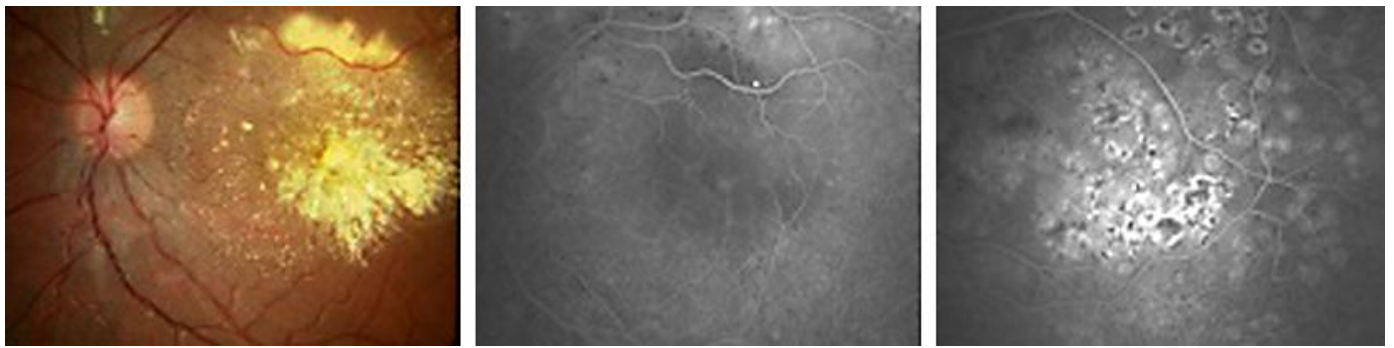

Fig. 3. Fluorescein angiography 4 months after treatment.

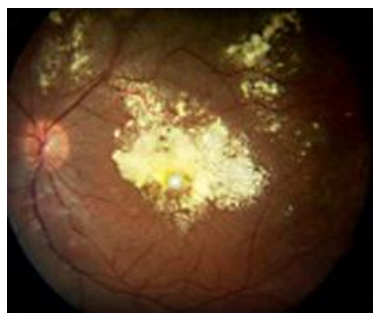

Fig. 4. Retinography 1 year after treatment, showing macular fibrosis.

\section{References}

1 Coats G: Forms of retinal disease with massive exudation. Roy London Ophthal Hosp Rep 1908;17:440525.

-2 Álvarez-Rivera LG, Abraham-Marín ML, Flores-Orta HJ, Mayorquín-Ruiz M, Cortés-Luna CF: Coat's disease treated with bevacizumab (Avastin) (in Spanish). Arch Soc Esp Oftalmol 2008;83:329-331.

-3 Smithen L, Brown G, Brucker A, Yanuzzi L, Klais C, Spaide R: Coats disease diagnosed in adulthood. Ophthalmology 2005;112:1072-1078.

-4 Andonegui J, Aranguren M, Berástegui L: Coats disease of adult onset. Arch Soc Esp Oftalmol 2008;83:117-120.

-5 Shields J, Shields C, Honavar S, Demirci H: Clinical variations and complications of Coats disease in 150 cases: the 2000 Sandford Gifford Memorial Lecture. Am J Ophthalmol 2001;131:561-571.

6 Kim J, Park KH, Woo SJ: Combined photodynamic therapy and intravitreal bevacizumab injection for the treatment of adult Coats disease: a case report. Korean J Ophthalmol 2010;24:374-376. 
-7 Cakir M, Cekiç 0, Yilmaz 0: Combined intravitreal bevacizumab and triamcinolone injection in a child with Coats disease. J AAPOS 2008;12:309-311.

8 Harris G: Coats disease, diagnosis and treatment. Can J Ophthalmol 1970;5:311-319.

-9 Jun JH, Kim YC, Kim KS: Resolution of severe macular edema in adult Coats disease with intravitreal triamcinolone and bevacizumab injection. Korean J Ophthalmol 2008;22:190-193.

10 Do DV, Haller JÁ: Coats disease; in Ryan SJ (ed): Retina. China, Elsevier Mosby, 2004, pp 1417-1423.

11 Vázquez-Pulido N, Laliena Santamaría JL, Del Buey Sayas MA, Burdeus Gómez R, Osan Tello M: Adultonset of recurrent Coats disease. Arch Soc Esp Oftalmol 2007;82:555-558.

12 Shields JA, Shields CL, Honavar S, et al: Classification and management of Coats disease: the 2000 Proctor Lecture. Am J Ophthalmol 2001;131:572-583.

13 Entezari M, Ramezani A, Safavizadeh L, Bassirnia N: Resolution of macular edema in Coats' disease with intravitreal bevacizumab. Indian J Ophthalmol 2010;58:80-82. 